\title{
The mechanics of short rod-like molecules in tension
}

\author{
Prashant K. Purohit ${ }^{1, *}$, Mark E. Arsenault ${ }^{1}$, Yale Goldman ${ }^{2}$ and Haim H. Bau ${ }^{1}$ \\ ${ }^{1}$ Mechanical Engineering and Applied Mechanics, ${ }^{2}$ Department of Physiology, \\ University of Pennsylvania, Philadelphia, PA 19104, USA.
}

Keywords: Fluctuating rods, force-extension, Brownian motion.

${ }^{*}$ Corresponding Author, email: purohit@seas.upenn.edu

\begin{abstract}
The rapid development of single molecule experimental techniques in the last two decades has made it possible to accurately measure the force-extension response as well as the transverse fluctuations of individual rod-like macromolecules. This information is used in conjunction with a statistical mechanical model based on the treatment of the molecule as a fluctuating elastic rod to extract its bending and extension moduli. The models most commonly used to interpret the experimental data assume that the magnitude of the Brownian fluctuations are independent of the length of the macromolecule, an assumption that holds only in the asymptotic limit of infinitely long rods, and is violated in most experiments. As an alternative, we present a theoretical treatment of a finite length, fluctuating rod and determine its mechanical behavior by measuring the transverse Brownian fluctuations under the action of large stretching forces. to validate of our thoery, we have applied our methods to an experiment on short actin filaments whose force-extension relation is difficult to measure, but whose transverse deflections can be captured by current microscopy techniques. An important consequence of the short contour lengths is that the boundary conditions applied in the experiment affect the fluctuations and can no longer be neglected as is commonly done when interpreting data from force-extension measurements. Our theoretical methods account for boundary conditons and can therefore be deployed in conjuction with forceextension measurements to obtain detailed information about the mechanical response of rod-like macromolecules.
\end{abstract}

\section{Introduction}

The development of single molecule visualization and manipulation techniques in the last two decades has propelled mechanics into the realms of molecular biophysics. It is now possible to exert known forces on biomolecules in the midst of chemical reactions[1] and observe the consequences at the nanometer scale. This has led to the realization that the mechanical properties of individual molecules which determine their response to mechanical stimuli are important players in the overall biochemistry. The technique most commonly employed to determine the mechanical properties of macromolecules is their force-extension response[2]. This technique has been used to determine the bending moduli of single- and double-stranded DNA[3] and RNA[4, 5] as well as proteins such as titin[6], tenascin[7] and spectrin[8]. The bending moduli of filamentous macromolecules such as actin and microtubules have also been determined, albeit using different techniques $[9,10]$. 
The majority of force-extension measurements on macromolecules are carried out in three different types of apparatus - optical tweezers, magnetic tweezers, and the atomic force microscope (AFM). Optical tweezers utilize the restoring forces exerted by light refracting through a spherical bead to manipulate attached macromolecules. A typical arrangement is to attach a filamentous molecule, such as, double-stranded DNA (dsDNA)[4], and actin[10], several micrometers in length, to polystyrene beads at each end, which are subsequently trapped in a laser beam. See figure 1(a). A known force (usually in the pN range) is exerted on the beads and the resulting distance between them is measured. The spherical beads are free to rotate since conventional laser traps do not exert moments. On the other hand, paramagnetic beads trapped by magnetic fields[11] or optical beads with anisotropic properties[12] (see figure 1(c)) can exert both forces and moments on the attached macromolecules. Such an apparatus has been used to study plectoneme formation in dsDNA[13, 14] and also to extract the twisting modulus of dsDNA by measuring how the force-extension behavior changes[14, 15] under constraints on the total linking number. Finally, the AFM is typically used to exert forces in the hundreds of $\mathrm{pN}$ range and has been used mostly to study the unfolding of proteins[16].

The force-extension data emerging from all these different types of apparatuses are most commonly interpreted using a statistical mechanical theory of Marko and Siggia[17] and Odijk[18]. The theory is valid for long molecules and is insensitive to the different types of boundary conditions imposed by different apparatus. Hence, a consequence of the use of this theory for interpretation of all manner of force-extension measurements (irrespective of the length of the molecule and testing apparatus) is that the mechanical properties are inferred to be functions of the contour length(see [19]). Ideally, we expect the mechanical properties to be independent of the length since they are intrinsic to the material. Furthermore, the end-to-end extension of a molecule is an observable that neglects inhomogeneities along the contour of the molecule (such as the folded vs. unfolded regions of a protein) and leads to the assignment of a single average property to the entire molecule. By augmenting extension measurements with measurements of transverse fluctuations, it may be possible to obtain local mechanical properties.

This paper represents an effort to infer mechanical behavior of filamentous macromolecules using a different observable - the magnitude of transverse Brownian fluctuations as a function of the position along the filament. We first develop a statistical mechanical theory that accounts for (i) the finite contour length of the filamentous macromolecule, and (ii) a variety of boundary conditions that can be applied to it. We then obtain expressions for the forceextension response as well as the transverse Brownian fluctuations as functions of the contour length and the applied boundary conditions. Some of our results are similar to those of two recent studies[19, 20] which also account for finite length effects and different boundary conditions of the testing apparatuses. However, our methods are different from both of these studies which rely on path integral techniques for semi-flexible polymers. We rely on the application of the equipartition theorem of statistical mechanics to derive our expressions, and hence our results are valid only when the deformations of the filament are not too large. On the other hand, path integral techniques can be used to study polymer conformations even under large deformations[17]. We use our theory to interpret data from an experiment designed to capture the transverse fluctuations of short actin filaments suspended across a gap. The filaments are trapped using AC dielectrophoresis[21, 22] and tracked in real time 
with a CCD camera. The emerging data are fit excellently by our theory with the tension being the only adjustible parameter.

Unlike the force-extension measurement, our method which is based on measuring transverse Brownian fluctuations, has the potential to capture local inhomogeneities. For instance, we expect that the transverse fluctuations of a filament will be large in regions where the bending modulus is small and vice-versa. When the measurements differ significantly from the values predicted by analytical expressions for homogeneous bending, a likely cause could be the presence of inhomogeneities. A conventional force-extension measurement will not be able to furnish such information. Furthermore, a method of interrogation based on transverse Brownian fluctuations would be able to distinguish between the response of the macromolecule under different types of boundary conditions. Finally, even with the same boundary conditions, we expect that the transverse fluctuations of a filamentous macromolecule will be larger for longer lengths, and so the contour length dependence can be properly accounted for. This would be advantageous for filaments with long persistence lengths for which it is difficult to design experiments consistent with the assumptions of asymptotic theories that assume a contour length much larger than the persistence length.

\section{Theory}

We consider a linearly elastic, isotropic, homogeneous, inextensible and unshearable rod whose center-line is parameterized by the position vector $\mathbf{r}(s)=r_{1}(s) \mathbf{e}_{1}+r_{2}(s) \mathbf{e}_{2}+z(s) \mathbf{e}_{3}$, where $s$ is the arc-length. The unit vector triad $\left[\begin{array}{lll}\mathbf{e}_{1} & \mathbf{e}_{2} & \mathbf{e}_{3}\end{array}\right]$ represents the laboratory coordinate frame. At each point $s$ along the rod, we have a material frame with a triad of orthogonal unit vectors $\left[\begin{array}{lll}\mathbf{d}_{1} & \mathbf{d}_{2} & \mathbf{d}_{3}\end{array}\right]$, such that $\mathbf{d}_{3}(s)=\frac{d \mathbf{r}}{d s}=t_{1}(s) \mathbf{e}_{1}+t_{2}(s) \mathbf{e}_{2}+t_{3}(s) \mathbf{e}_{3}$ is the tangent to the rod. The curvature vector $\kappa(s)=\kappa_{1}(s) \mathbf{d}_{1}(s)+\kappa_{2}(s) \mathbf{d}_{2}(s)+\kappa_{3}(s) \mathbf{d}_{3}(s)$ is defined through

$$
\frac{d \mathbf{d}_{i}}{d s}=\kappa \times \mathbf{d}_{i}
$$

The torque

$$
\mathbf{M}=K_{b} \kappa_{1} \mathbf{d}_{1}+K_{b} \kappa_{2} \mathbf{d}_{2}+K_{t} \kappa_{3} \mathbf{d}_{3}
$$

is a linear function of the curvature vector $\kappa$. In the above, $K_{b}$ is the bending modulus and $K_{t}$ is the twisting modulus. A constant tension $\mathbf{F}=F \mathbf{e}_{3}$ and a constant twisting moment $\tau$ are applied to the rod (for instance, in a magnetic tweezer set-up) with the goal of measuring the corresponding end-to-end extension. When the extension of the molecule is controlled (as in an AFM) then $\mathbf{F}$ is interpreted as a Lagrange multiplier enforcing the constraint $\left|\frac{d \mathbf{r}}{d s}\right|=1$. The potential energy functional for such a rod can be written as

$$
E=\int_{-L / 2}^{L / 2} \frac{K_{b}}{2}\left(\kappa_{1}^{2}+\kappa_{2}^{2}\right) d s+\int_{-L / 2}^{L / 2} \frac{K_{t}}{2} \kappa_{3}^{2} d s-\int_{-L / 2}^{L / 2} \mathbf{F} \cdot \mathbf{d}_{3} d s-2 \pi \tau L k
$$

where $L k$ is the total linking number. The statistical mechanics of such a rod is rather cumbersome and was described in detail by Moroz and Nelson[23]. We will adopt a simpler approach, which is a modified version of the worm-like chain model proposed by Marko and Siggia[17] for stretching DNA. The major assumption is that the applied tension $F$ is 
sufficiently large so that the tangent to the $\operatorname{rod} \mathbf{d}_{3}(s)$ fluctuates around the average value of $\mathbf{e}_{3}$, which is coaligned with the force $\mathbf{F}$. We also assume that there are no torsional constraints on the rod so that the second and fourth term on the right hand side of (3) can be dropped. As a consequence of these two assumptions, we have

$$
\mathbf{d}_{3}(s) \cdot \mathbf{e}_{3}=t_{3}(s)=\cos \theta(s)=\sqrt{1-\left(t_{1}^{2}+t_{2}^{2}\right)} \approx 1-\frac{1}{2}\left(t_{1}^{2}+t_{2}^{2}\right)
$$

and

$$
\kappa_{1}^{2}(s)+\kappa_{2}^{2}(s)=\left(\frac{d \theta}{d s}\right)^{2}=\left|\frac{d t_{1}}{d s} \mathbf{e}_{1}+\frac{d t_{2}}{d s} \mathbf{e}_{2}\right|^{2},
$$

where $\theta(s)$ is the angle between the tangent $\mathbf{d}_{3}(s)$ and the $\mathbf{e}_{3}$ axis. Note that there are no restrictions on the magnitude of the displacements, but the analysis is restricted to small deviations of the tangent vector $\mathbf{d}_{3}(s)$ from $\mathbf{e}_{3}{ }^{1}$. In the literature on the mechanics of rods, approximation (4) corresponds to small strains and moderate rotations (see [24, 25] and references therein). Under the above conditions, bending, twisting, and extensional deformations of a rod are coupled. This is in contrast to a small strain, small rotation theory where $\mathbf{d}_{3} \cdot \mathbf{e}_{3}=\frac{d z}{d s}=t_{3}(s) \approx 1$ and $z(s) \approx s$. In such a theory, bending, twisting, and extension are independent of each other. Furthermore, $z(s) \approx s$ implies that the displacements are also small.

The potential energy functional for the small strain moderate rotation theory can be written as

$$
E=\int_{-L / 2}^{L / 2} \frac{K_{b}}{2}\left|\frac{d \mathbf{t}_{\perp}}{d s}\right|^{2} d s+\int_{-L / 2}^{L / 2} \frac{F}{2}\left|\mathbf{t}_{\perp}\right|^{2} d s-F L
$$

where we have introduced the notation $\mathbf{t}_{\perp}(s)=t_{1}(s) \mathbf{e}_{1}+t_{2}(s) \mathbf{e}_{2}$. Next, we expand $\mathbf{t}_{\perp}(s)$ in an appropriate complete set of orthogonal basis functions:

$$
\mathbf{t}_{\perp}(s)=\sum_{n=1}^{\infty} \mathbf{A}_{n} f_{n}^{o}(s)+\sum_{m=1}^{\infty} \mathbf{B}_{m} f_{m}^{e}(s)
$$

where $n$ and $m$ are mode numbers, $f_{n}^{o}(s)$ and $f_{m}^{e}(s)$ are, respectively, odd and even basis functions, $\mathbf{A}_{n}=A_{n 1} \mathbf{e}_{1}+A_{n 2} \mathbf{e}_{2}$, and $\mathbf{B}_{m}=B_{m 1} \mathbf{e}_{1}+B_{m 2} \mathbf{e}_{2}$. We will consider explicitly the three cases depicted in figure 1: (a) hinged-hinged filament, (b) clamped-clamped filament, and (c) partially clamped filament. In each of these cases, there are four boundary conditions to be satisfied. The following representation of $\mathbf{t}_{\perp}(s)$ is appropriate for such circumstances:

$$
\mathbf{t}_{\perp}(s)=\sum_{n=1}^{\infty} \mathbf{A}_{n}\left[\sin \left(k_{o}^{n} s\right)+\alpha_{n} \sinh \left(k_{o}^{n} s\right)\right]+\sum_{m=1}^{\infty} \mathbf{B}_{m}\left[\cos \left(k_{e}^{m} s\right)+\beta_{m} \cosh \left(k_{e}^{m} s\right)\right],
$$

where $k_{o}^{n}, k_{e}^{m}, \alpha_{n}$ and $\beta_{m}$ are constants that are determined by the boundary conditions. Substituting the series expansion of $\mathbf{t}_{\perp}(s)$ into (6), assuming that the modes are independent ${ }^{2}$, and taking averages in a canonical ensemble, we find that the average energy takes

\footnotetext{
${ }^{1}$ For instance, when $\theta=\frac{\pi}{6}$ we make an error of $1 \%$ by using the approximate expression $(4)$ for $\cos \theta(s)$. When $\theta=\frac{\pi}{4}$, the error is $6 \%$.

${ }^{2} \mathbf{A}_{n}$ and $\mathbf{B}_{m}$ are not independent for the problem in figure 1(c).
} 

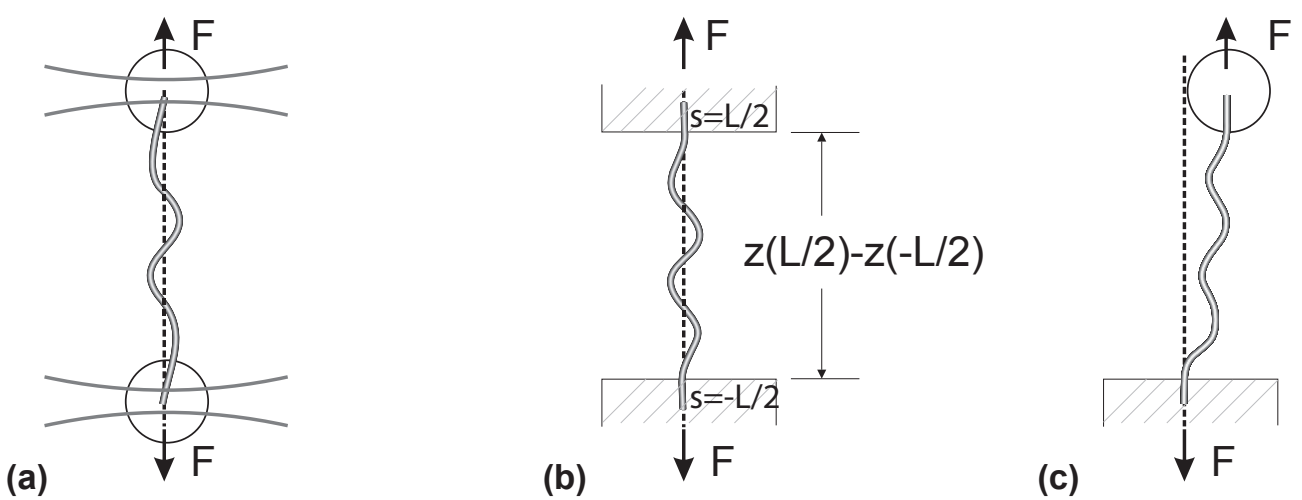

Figure 1: Set-ups for measuring the force vs. extension behavior of macromolecules. (a) Hinged-hinged condition. Both ends are attached to beads which are held in optical traps that can exert forces but not moments. Hence the curvatures at the ends are constrained but not the slopes. The trap does not allow transverse displacements. (b) Clamped-clamped condition. The slopes and transverse displacements are constrained at both ends. (c) Partially clamped condition. One end of the macromolecule is secured to a cover-slip while the other end is attached to a bead in a magnetic or optical trap which ensures that the slope is held constant, but transverse displacements are allowed.

the form

$$
\langle E\rangle=\sum_{n=1}^{\infty}\left\langle\left|\mathbf{A}_{n}\right|^{2}\right\rangle g_{n}^{o}\left(K_{b}, F, L\right)+\sum_{m=1}^{\infty}\left\langle\left|\mathbf{B}_{m}\right|^{2}\right\rangle g_{m}^{e}\left(K_{b}, F, L\right)-F L
$$

where $g_{n}^{o}\left(K_{b}, F, L\right)$ and $g_{m}^{e}\left(K_{b}, F, L\right)$ are functions that depend on the boundary conditions as well as on the particular values of the bending modulus $K_{b}$, force $F$, and the length $L$ of the macromolecule. In the above, $\langle\cdot\rangle$ represents an average in the canonical ensemble which requires constant length, constant temperature, and no mass transfer. With the exception of case (c) (see figure 1), there are no cross-terms with coefficients $\mathbf{A}_{n} \cdot \mathbf{B}_{m}$ since the domain of integration is $\left[-\frac{L}{2}, \frac{L}{2}\right]$ and we have chosen a set of even and odd basis functions. Moreover, $\left\langle\mathbf{A}_{n} \cdot \mathbf{A}_{n+i}\right\rangle=\left\langle\mathbf{B}_{m} \cdot \mathbf{B}_{m+i}\right\rangle=0$ for $i \neq 0$, since the modes are independent.

According to the equipartition theorem of statistical mechanics, every quadratic mode in equilibrium has an energy $\frac{k_{B} T}{2}$, where $k_{B}$ is the Boltzmann constant and $T$ is the absolute temperature. This allows us to determine the mean squared-amplitudes $\left\langle\left|\mathbf{A}_{n}\right|^{2}\right\rangle$ and $\left\langle\left|\mathbf{B}_{m}\right|^{2}\right\rangle$ of each mode which can then be used to determine the force-extension relation and the magnitude of the transverse fluctuations along the rod using the relations

$$
z(s)-z\left(-\frac{L}{2}\right)=\int_{-L / 2}^{s} \cos \theta d s \approx \int_{-L / 2}^{s}\left(1-\frac{\left|\mathbf{t}_{\perp}\right|^{2}}{2}\right) d s=\left(s+\frac{L}{2}\right)-\frac{1}{2} \int_{-L / 2}^{s}\left|\mathbf{t}_{\perp}\right|^{2} d s
$$

and

$$
\left|\mathbf{r}_{\perp}(s)-\mathbf{r}_{\perp}\left(-\frac{L}{2}\right)\right|^{2}=\int_{-L / 2}^{s} \int_{-L / 2}^{s} \mathbf{t}_{\perp}(u) \cdot \mathbf{t}_{\perp}(v) d u d v
$$

where we have used (4) and defined

$$
\mathbf{r}_{\perp}(s)=\mathbf{r}_{\perp}(0)+\int_{0}^{s} \mathbf{t}_{\perp}(s)=r_{1}(s) \mathbf{e}_{1}+r_{2}(s) \mathbf{e}_{2} .
$$


We illustrate this procedure for the three special cases depicted in figure 1 . Of these, the clamped-clamped condition is used in our experiments while the other two are commonly used for most force-extension measurements.

Case (a) Hinged-hinged filament: When the ends of the filament are attached to beads in an optical trap, we require $\frac{d \mathbf{t}_{\perp}}{d s}=\mathbf{0}$ at $s= \pm \frac{L}{2}$ since optical traps can only apply forces on a bead but not moments. We also require $\mathbf{r}_{\perp}\left(-\frac{L}{2}\right)=\mathbf{r}_{\perp}\left(\frac{L}{2}\right)=\mathbf{0}$ since the trap constrains the transverse motion of the beads. Starting from (8) and applying the above boundary conditions, we find that $\alpha_{n}=0$ for all $n$ and $\beta_{m}=0$ for all $m$, so that we are left with

$$
\mathbf{t}_{\perp}(s)=\sum_{n=1}^{\infty} \mathbf{A}_{n} \sin \frac{(2 n-1) \pi s}{L}+\sum_{m=1}^{\infty} \mathbf{B}_{m} \cos \frac{2 m \pi s}{L} .
$$

Using (6) the average energy can be written as

$$
\langle E\rangle=\sum_{n=1}^{\infty}\left(\frac{K_{b}}{4} \frac{(2 n-1)^{2} \pi^{2}}{L}+\frac{F L}{4}\right)\left\langle\left|\mathbf{A}_{n}\right|^{2}\right\rangle+\sum_{m=1}^{\infty}\left(\frac{K_{b}}{4} \frac{4 m^{2} \pi^{2}}{L}+\frac{F L}{4}\right)\left\langle\left|\mathbf{B}_{m}\right|^{2}\right\rangle-F L .
$$

The equipartition theorem then gives

$$
\left\langle\left|\mathbf{A}_{n}\right|^{2}\right\rangle=\frac{4 k_{B} T L}{K_{b}(2 n-1)^{2} \pi^{2}+F L^{2}} \quad \text { and } \quad\left\langle\left|\mathbf{B}_{m}\right|^{2}\right\rangle=\frac{4 k_{B} T L}{4 K_{b} m^{2} \pi^{2}+F L^{2}}
$$

where we have accounted for the fact that each $n$ and $m$ term contains two modes, i.e., the corresponding energy is $k_{B} T$. The average extension $\left\langle z(s)-z\left(-\frac{L}{2}\right)\right\rangle$ as a function of the position along the filament may now be computed using (10) and (15) as follows:

$$
\begin{aligned}
\left\langle z(s)-z\left(-\frac{L}{2}\right)\right\rangle= & s+\frac{L}{2}-\frac{1}{2} \int_{-L / 2}^{s} \sum_{n=1}^{\infty}\left\langle\left|\mathbf{A}_{n}\right|^{2}\right\rangle \sin ^{2} \frac{(2 n-1) \pi s}{L} d s \\
& -\frac{1}{2} \int_{-L / 2}^{s} \sum_{m=1}^{\infty}\left\langle\left|\mathbf{B}_{m}\right|^{2}\right\rangle \cos ^{2} \frac{2 m \pi s}{L} d s \\
= & s+\frac{L}{2}-\sum_{p=1}^{\infty} \frac{k_{B} T L}{K_{b} p^{2} \pi^{2}+F L^{2}}\left[s+\frac{L}{2}+\frac{(-1)^{p} L}{2 p \pi} \sin \frac{2 p \pi s}{L}\right] \\
= & \left(s+\frac{L}{2}\right)\left[1-\frac{k_{B} T}{2 \sqrt{K_{b} F}}\left(\operatorname{coth}\left(L \sqrt{\frac{F}{K_{b}}}\right)-\frac{1}{L} \sqrt{\frac{K_{b}}{F}}\right)\right] \\
& -\frac{k_{B} T}{4 F}\left[\frac{\sinh \left(2 s \sqrt{\frac{F}{K_{b}}}\right)}{\sinh \left(L \sqrt{\frac{F}{K_{b}}}\right)}-\frac{2 s}{L}\right] .
\end{aligned}
$$

In particular, the average end-to-end extension

$$
\left\langle z\left(\frac{L}{2}\right)-z\left(-\frac{L}{2}\right)\right\rangle=L-\frac{k_{B} T L}{2 \sqrt{K_{b} F}}\left(\operatorname{coth}\left(L \sqrt{\frac{F}{K_{b}}}\right)-\frac{1}{L} \sqrt{\frac{K_{b}}{F}}\right) .
$$


This expression summarizes the force-extension relation for a macromolecule held in an optical trap. We observe that for a hinged-hinged macromolecule, $\left[1-\frac{\left\langle z\left(\frac{L}{2}\right)-z\left(-\frac{L}{2}\right)\right\rangle}{L}\right] \rightarrow \frac{k_{B} T}{2 \sqrt{K_{b} F}}$ in the limit of $L \sqrt{\frac{F}{K_{b}}} \rightarrow \infty$, which is the result of Marko and Siggia[17]. Interestingly, (17) is identical to an expression derived by Hori et al.[20] for a different set of boundary conditions (which are the same as our partially-clamped case depicted in figure 1(c)).

Although the theory is not strictly valid for small $F$, it is still worthwhile to investigate the asymptotic behavior as $F \rightarrow 0$. We find, in the limit of $F \rightarrow 0$,

$$
\frac{\left\langle z\left(\frac{L}{2}\right)-z\left(-\frac{L}{2}\right)\right\rangle}{L} \approx 1-\frac{1}{6} \frac{L}{L_{p}}+\frac{1}{90}\left(\frac{F L^{2}}{K_{b}}\right) \frac{L}{L_{p}}+\ldots
$$

where $L_{p}=\frac{K_{b}}{k_{B} T}$ is the persistence length. In the absence of tension, the estimated average end-to-end extension is somewhat smaller than the contour length due to thermal fluctuations.

The excursions of the macromolecule in the planes with normal $\mathbf{e}_{3}$ can be similarly computed using (11) and (15) to give the result

$$
\begin{aligned}
\left\langle\left|\mathbf{r}_{\perp}(s)-\mathbf{r}_{\perp}\left(-\frac{L}{2}\right)\right|^{2}\right\rangle= & \sum_{n=1}^{\infty} \frac{4 k_{B} T L}{K_{b}(2 n-1)^{2} \pi^{2}+F L^{2}} \frac{L^{2}}{(2 n-1)^{2} \pi^{2}} \cos ^{2} \frac{(2 n-1) \pi s}{L} \\
& +\sum_{m=1}^{\infty} \frac{4 k_{B} T L}{4 K_{b} m^{2} \pi^{2}+F L^{2}} \frac{L^{2}}{4 m^{2} \pi^{2}} \sin ^{2} \frac{2 m \pi s}{L} .
\end{aligned}
$$

Witness that the series decays rapidly as the coefficients behave like $m^{-4}$. The series may be summed to give

$$
\left\langle\left|\mathbf{r}_{\perp}(s)-\mathbf{r}_{\perp}\left(-\frac{L}{2}\right)\right|^{2}\right\rangle=\frac{2 k_{B} T}{F L}\left[\left(\frac{L}{2}+s\right)\left(\frac{L}{2}-s\right)-L \sqrt{\frac{K_{b}}{F}}\left(\frac{\sinh ^{2}\left(\frac{L}{2} \sqrt{\frac{F}{K_{b}}}\right)-\sinh ^{2}\left(s \sqrt{\frac{F}{K_{b}}}\right)}{\sinh \left(L \sqrt{\frac{F}{K_{b}}}\right)}\right)\right]
$$

Not surprisingly, $\left\langle\left|\mathbf{r}_{\perp}\left(\frac{L}{2}\right)-\mathbf{r}_{\perp}\left(-\frac{L}{2}\right)\right|^{2}\right\rangle=0$, as expected for hinged-hinged boundary conditions. Also, when $K_{b}=0$ we recover an expression similar to the one derived by van Lear and Uhlenbeck[26] for a vibrating string. Finally, it is evident that the transverse fluctuations of the macromolecule are large for large $L$ and small for large $K_{b}$ and $F$.

Case (b) Clamped-clamped filament: Clamping the ends of the macromolecule implies that $\mathbf{t}_{\perp}\left(-\frac{L}{2}\right)=\mathbf{t}_{\perp}\left(\frac{L}{2}\right)=0$ and $\mathbf{r}_{\perp}\left(-\frac{L}{2}\right)=\mathbf{r}_{\perp}\left(\frac{L}{2}\right)=\mathbf{0}$. Starting from (8) and imposing these boundary conditions, we see that $\mathbf{t}_{\perp}(s)$ can be expressed as follows:

$$
\mathbf{t}_{\perp}(s)=\sum_{n=1}^{\infty} \mathbf{A}_{n}\left(\frac{\cosh \left(k_{n}^{o} s\right)}{\sinh \left(\frac{k_{n}^{o} L}{2}\right)}-\frac{\cos \left(k_{n}^{o} s\right)}{\sin \left(\frac{k_{n}^{o} L}{2}\right)}\right)+\sum_{m=1}^{\infty} \mathbf{B}_{m}\left(\frac{\sinh \left(k_{m}^{e} s\right)}{\cosh \left(\frac{k_{m}^{e} L}{2}\right)}+\frac{\sin \left(k_{m}^{e} s\right)}{\cos \left(\frac{k_{m}^{e} L}{2}\right)}\right),
$$

where $k_{n}^{o}$ are the roots of the equation $\tan \left(\frac{k_{n}^{o} L}{2}\right)-\tanh \left(\frac{k_{n}^{o} L}{2}\right)=0$ and $k_{m}^{e}$ are the roots of the equation $\tan \left(\frac{k_{m}^{e} L}{2}\right)+\tanh \left(\frac{k_{m}^{e} L}{2}\right)=0$. The first few roots are $k_{1}^{o} L \approx 7.8532, k_{2}^{o} L \approx 14.1373$, $\ldots, k_{n}^{o} L \approx(4 n+1) \frac{\pi}{2}$ and $k_{1}^{e} L \approx 4.7300, k_{2}^{e} L \approx 10.9956, \ldots, k_{m}^{o} L \approx(4 m-1) \frac{\pi}{2}$. 


\begin{tabular}{|c||c|c|c|c|c|}
\hline$\frac{F L_{p}}{k_{B} T}$ & $\left\langle\left|\mathbf{A}_{1}\right|^{2}\right\rangle \times 10^{4}$ & $\left\langle\left|\mathbf{A}_{2}\right|^{2}\right\rangle \times 10^{4}$ & $\left\langle\left|\mathbf{A}_{3}\right|^{2}\right\rangle \times 10^{4}$ & $\left\langle\left|\mathbf{A}_{4}\right|^{2}\right\rangle \times 10^{4}$ & $\left\langle\left|\mathbf{A}_{5}\right|^{2}\right\rangle \times 10^{4}$ \\
\hline \hline $10^{0}$ & 170.0 & 51.36 & 24.61 & 14.39 & 9.43 \\
\hline $10^{2}$ & 130.0 & 51.30 & 23.26 & 13.91 & 9.21 \\
\hline $10^{4}$ & 4.992 & 4.117 & 3.631 & 3.223 & 2.853 \\
\hline \hline$\frac{F L_{p}}{k_{B} T}$ & $\left\langle\left|\mathbf{B}_{1}\right|^{2}\right\rangle \times 10^{4}$ & $\left\langle\left|\mathbf{B}_{2}\right|^{2}\right\rangle \times 10^{4}$ & $\left\langle\left|\mathbf{B}_{3}\right|^{2}\right\rangle \times 10^{4}$ & $\left\langle\left|\mathbf{B}_{4}\right|^{2}\right\rangle \times 10^{4}$ & $\left\langle\left|\mathbf{B}_{5}\right|^{2}\right\rangle \times 10^{4}$ \\
\hline \hline $10^{0}$ & 460.0 & 84.89 & 34.38 & 18.49 & 11.52 \\
\hline $10^{2}$ & 280.0 & 71.79 & 31.84 & 17.70 & 11.21 \\
\hline $10^{4}$ & 6.88 & 4.45 & 3.86 & 3.42 & 3.03 \\
\hline
\end{tabular}

Table 1: The mean square amplitudes $\left\langle\left|\mathbf{A}_{n}\right|^{2}\right\rangle$ and $\left\langle\left|\mathbf{B}_{m}\right|^{2}\right\rangle$ decay with increasing mode numbers $n$ and $m$. Here we use $\frac{L}{L_{p}}=0.5$ and assume clamped-clamped boundary conditions.

Analogous to the hinged-hinged case, we use the equipartition theorem to get

$$
\begin{aligned}
\frac{4 k_{B} T}{\left\langle\left|\mathbf{A}_{n}\right|^{2}\right\rangle}= & K_{b} k_{n}^{o}\left[\frac{\sinh \left(k_{n}^{o} L\right)-k_{n}^{o} L}{\sinh ^{2}\left(\frac{k_{n}^{o} L}{2}\right)}+\frac{-\sin \left(k_{n}^{o} L\right)+k_{n}^{o} L}{\sin ^{2}\left(\frac{k_{n}^{o} L}{2}\right)}\right] \\
& +\frac{F}{k_{n}^{o}}\left[\frac{\sinh \left(k_{n}^{o} L\right)+k_{n}^{o} L}{\sinh ^{2}\left(\frac{k_{n}^{o} L}{2}\right)}+\frac{\sin \left(k_{n}^{o} L\right)+k_{n}^{o} L}{\sin ^{2}\left(\frac{k_{n}^{o} L}{2}\right)}-8 \operatorname{coth}\left(\frac{k_{n}^{o} L}{2}\right)\right],
\end{aligned}
$$

and

$$
\begin{aligned}
\frac{4 k_{B} T}{\left\langle\left|\mathbf{B}_{m}\right|^{2}\right\rangle}= & K_{b} k_{m}^{e}\left[\frac{\sinh \left(k_{m}^{e} L\right)+k_{m}^{e} L}{\cosh ^{2}\left(\frac{k_{m}^{e} L}{2}\right)}+\frac{\sin \left(k_{m}^{e} L\right)+k_{m}^{e} L}{\cos ^{2}\left(\frac{k_{m}^{e} L}{2}\right)}\right] \\
& +\frac{F}{k_{m}^{e}}\left[\frac{\sinh \left(k_{m}^{e} L\right)-k_{m}^{e} L}{\cosh ^{2}\left(\frac{k_{m}^{e} L}{2}\right)}+\frac{-\sin \left(k_{m}^{e} L\right)+k_{m}^{e} L}{\cos ^{2}\left(\frac{k_{m}^{e} L}{2}\right)}-8 \tanh \left(\frac{k_{m}^{e} L}{2}\right)\right] .
\end{aligned}
$$

A few values of $\left\langle\left|\mathbf{A}_{n}\right|^{2}\right\rangle$ and $\left\langle\left|\mathbf{B}_{m}\right|^{2}\right\rangle$ are given in table 1 for various tensions.

Using (10), (11), (22) and (23), we compute the extension $\left\langle z(s)-z\left(-\frac{L}{2}\right)\right\rangle$ and the magnitude of the transverse fluctuations $\left\langle\left|\mathbf{r}_{\perp}(s)-\mathbf{r}_{\perp}\left(-\frac{L}{2}\right)\right|^{2}\right\rangle$ as functions of the position along the macromolecule. The expression for $\left\langle z(s)-z\left(-\frac{L}{2}\right)\right\rangle$ is lengthy and will not be reproduced here but the end-to-end extension is given by the following series expression:

$$
\begin{aligned}
\left\langle z\left(\frac{L}{2}\right)-z\left(-\frac{L}{2}\right)\right\rangle & =L-\frac{1}{2} \sum_{n=1}^{\infty}\left\langle\left|\mathbf{A}_{n}\right|^{2}\right\rangle \frac{1}{k_{n}^{o}}\left[\frac{\sinh \left(k_{n}^{o} L\right)+k_{n}^{o} L}{2 \sinh ^{2}\left(\frac{k_{n}^{o} L}{2}\right)}+\frac{\sin \left(k_{n}^{o} L\right)+k_{n}^{o} L}{2 \sin ^{2}\left(\frac{k_{n}^{o} L}{2}\right)}-4 \operatorname{coth}\left(\frac{k_{n}^{o} L}{2}\right)\right] \\
& -\frac{1}{2} \sum_{m=1}^{\infty}\left\langle\left|\mathbf{B}_{m}\right|^{2}\right\rangle \frac{1}{k_{m}^{e}}\left[\frac{\sinh \left(k_{m}^{e} L\right)-k_{m}^{e} L}{2 \cosh ^{2}\left(\frac{k_{m}^{e} L}{2}\right)}+\frac{-\sin \left(k_{m}^{e} L\right)+k_{m}^{e} L}{2 \cos ^{2}\left(\frac{k_{m}^{e} L}{2}\right)}-4 \tanh \left(\frac{k_{m}^{e} L}{2}\right)\right]
\end{aligned}
$$

Numerical calculations indicate that the series (24) is equivalent to the closed form expression derived by Hori et al.[20] for the same boundary conditions (which they refer to as "axis clamping") using path integral techniques. 
The magnitude of the transverse fluctuations as a function of the position is similarly calculated as a series expression and is given below:

$$
\begin{aligned}
\left\langle\left|\mathbf{r}_{\perp}(s)-\mathbf{r}_{\perp}\left(-\frac{L}{2}\right)\right|^{2}\right\rangle & =\sum_{n=1}^{\infty}\left\langle\left|\mathbf{A}_{n}\right|^{2}\right\rangle\left(\frac{1}{k_{n}^{o}}\right)^{2}\left(\frac{\sinh \left(k_{n}^{o} s\right)}{\sinh \left(\frac{k_{n}^{o} L}{2}\right)}-\frac{\sin \left(k_{n}^{o} s\right)}{\sin \left(\frac{k_{n}^{o} L}{2}\right)}\right)^{2} \\
& +\sum_{m=1}^{\infty}\left\langle\left|\mathbf{B}_{m}\right|^{2}\right\rangle\left(\frac{1}{k_{m}^{e}}\right)^{2}\left(\frac{\cosh \left(k_{m}^{e} s\right)}{\cosh \left(\frac{k_{m}^{e} L}{2}\right)}-\frac{\cos \left(k_{m}^{e} s\right)}{\cos \left(\frac{k_{m}^{e} L}{2}\right)}\right)^{2}
\end{aligned}
$$

It is evident from the above expression that $\left\langle\left|\mathbf{r}_{\perp}\left(\frac{L}{2}\right)-\mathbf{r}_{\perp}\left(-\frac{L}{2}\right)\right|^{2}\right\rangle=0$ as expected for clampedclamped boundary conditions. The rate of decay of the coefficients $\left\langle\left|\mathbf{A}_{n}\right|^{2}\right\rangle$ and $\left\langle\left|\mathbf{B}_{m}\right|^{2}\right\rangle$ depends on the relative values of $F$ and $K_{b}$. In many cases, it is sufficient to retain the leading order terms.

Case (c) Partially clamped: When one end of the filament is clamped while the other is attached to a bead that constrains the tangent but can translate laterally, we set $\mathbf{t}_{\perp}\left(-\frac{L}{2}\right)=$ $\mathbf{t}_{\perp}\left(\frac{L}{2}\right)=\mathbf{0}, \mathbf{r}_{\perp}\left(-\frac{L}{2}\right)=\mathbf{0}$, and $\left.\frac{d^{2} \mathbf{t}_{\perp}}{d s^{2}}\right|_{s=L / 2}=\mathbf{0}^{3}$. Such a boundary condition can be realized by the use of optical traps capable of angular trapping $[12,13]$. The traps rely on anisotropic calcite microparticles and can constrain the tangent at the end while still allowing free rotation around the axis $\mathbf{e}_{3}$. In such a scenario, a routine calculation leads to

$$
\mathbf{t}_{\perp}(s)=\sum_{n=1}^{\infty} \mathbf{A}_{n}\left(\frac{\sinh \left(k_{n} s\right)}{\sinh \left(\frac{k_{n} L}{2}\right)}-\frac{\sin \left(k_{n} s\right)}{\sin \left(\frac{k_{n} L}{2}\right)}\right)+\sum_{n=1}^{\infty} \mathbf{B}_{n}\left(\frac{\cosh \left(k_{n} s\right)}{\cosh \left(\frac{k_{n} L}{2}\right)}-\frac{\cos \left(k_{n} s\right)}{\cos \left(\frac{k_{n} L}{2}\right)}\right),
$$

where $\mathbf{B}_{n}=-\mathbf{A}_{n}$ and $k_{n}$ are the roots of the equation $\tan \left(k_{n} L\right)+\tanh \left(k_{n} L\right)=0$. The first few roots are $k_{1} L=2.3650, k_{2} L=5.4978, \ldots, k_{n} L=(4 n-1) \frac{\pi}{4}$. Using the equipartition theorem, we obtain an expression for the mean-squared amplitudes $\left\langle\left|\mathbf{A}_{n}\right|^{2}\right\rangle$ as follows:

$$
\frac{k_{B} T}{\left\langle\left|\mathbf{A}_{n}\right|^{2}\right\rangle}=K_{b} k_{n}^{2} L\left[\frac{1}{\sinh ^{2}\left(k_{n} L\right)}+\frac{1}{\sin ^{2}\left(k_{n} L\right)}\right]+F L\left[\frac{1}{\sin ^{2}\left(k_{n} L\right)}-\frac{1}{\sinh ^{2}\left(k_{n} L\right)}+2 \cot \left(k_{n} L\right)\right] \text {. }
$$

Using (27), (10), and (11), we compute the end-to-end extension $\left\langle z\left(\frac{L}{2}\right)-z\left(-\frac{L}{2}\right)\right\rangle$ and the magnitude of the transverse fluctuations $\left\langle\left|\mathbf{r}_{\perp}(s)-\mathbf{r}_{\perp}\left(-\frac{L}{2}\right)\right|^{2}\right\rangle$ as functions of the position along the macromolecule. The results in the form of series expressions are given below:

$$
\left\langle z\left(\frac{L}{2}\right)-z\left(-\frac{L}{2}\right)\right\rangle=L\left[1-\sum_{n=1}^{\infty}\left\langle\left|\mathbf{A}_{n}\right|^{2}\right\rangle\left(\frac{1}{\sin ^{2}\left(k_{n} L\right)}-\frac{1}{\sinh ^{2}\left(k_{n} L\right)}+2 \cot \left(k_{n} L\right)\right)\right],
$$

and

$$
\left\langle\left|\mathbf{r}_{\perp}(s)-\mathbf{r}_{\perp}\left(-\frac{L}{2}\right)\right|^{2}\right\rangle=\sum_{n=1}^{\infty}\left\langle\left|\mathbf{A}_{n}\right|^{2}\right\rangle\left(\frac{1}{k_{n}}\right)^{2}\left(\frac{\cosh \left(k_{n} s\right)-\cosh \left(\frac{k_{n} L}{2}\right)}{\sinh \left(\frac{k_{n} L}{2}\right)}+\frac{\cos \left(k_{n} s\right)-\cos \left(\frac{k_{n} L}{2}\right.}{\sin \left(\frac{k_{n} L}{2}\right)}\right)^{2}
$$

\footnotetext{
${ }^{3}$ Moment equilibrium of an inextensible filament requires that $\frac{d \mathbf{M}}{d s}+\mathbf{d}_{3} \times \mathbf{F}=\mathbf{0}$ at all points $s$ (see [27]). At $s=L / 2$ we have $\mathbf{d}_{3}(L / 2)=\mathbf{e}_{3}$ which implies that $\mathbf{d}_{3} \times \mathbf{F}=\mathbf{0}$ since $\mathbf{F}=F \mathbf{e}_{3}$. As a result the appropriate natural boundary condition at $s=\frac{L}{2}$ is $\frac{d \mathbf{M}}{d s}=\mathbf{0}$. Since we assume that there are no torsional constraints, it follows that $K_{b} \frac{d^{2} \mathbf{t}_{\perp}}{d s^{2}}=\mathbf{0}$ at $s=L / 2$.
} 


$$
+\sum_{n=1}^{\infty}\left\langle\left|\mathbf{A}_{n}\right|^{2}\right\rangle\left(\frac{1}{k_{n}}\right)^{2}\left(\frac{\sinh \left(k_{n} s\right)+\sinh \left(\frac{k_{n} L}{2}\right)}{\cosh \left(\frac{k_{n} L}{2}\right)}-\frac{\sin \left(k_{n} s\right)+\sin \left(\frac{k_{n} L}{2}\right.}{\cos \left(\frac{k_{n} L}{2}\right)}\right)^{2}
$$

\section{Discussion}

The dependence of the transverse fluctuations $\left\langle\left|\mathbf{r}_{\perp}(s)-\mathbf{r}_{\perp}\left(-\frac{L}{2}\right)\right|^{2}\right\rangle$ on the position $s$ for each of the apparatuses depicted in figure 1 can be better understood by referring to figure 2(a). For the cases where we have series expressions, we have plotted the sums of the first 5 terms since the series converge rapidly. The transverse fluctuations of the partially clamped filament (figure 1(c)) are non-zero at the end held in the trap, since the bead is free to translate in any direction. For the hinged-hinged filament, the transverse fluctuations are zero at both ends. We contrast this with the clamped-clamped filament whose transverse fluctuations are also zero at both ends but the magnitudes of whose excursions are much smaller than those of the hinged-hinged filament at all locations along the contour. In other words, the clamped-clamped filament appears to be stiffer than the hinged-hinged filament even though the values of the bending modulus $K_{b}$, the tension $F$, and the contour length $L$ are the same. This is solely a result of the different boundary conditions.

The force-extension relation as well as the magnitude of the transverse fluctuations are also strongly dependent on the length of the macromolecule. This can be appreciated by appealing to figure 2(b) where we have plotted the normalized average end-to-end extension (with hinged-hinged boundary conditions) as a function of the normalized axial force for various values of the contour length $L$. The contour length is normalized with the persistence length $L_{p}=\frac{K_{b}}{k_{B} T}$ and the force is normalized with $\frac{k_{B} T}{L_{p}}$. The lower limit of the ordinate $\frac{\left\langle z\left(\frac{L}{2}\right)-z\left(-\frac{L}{2}\right)\right\rangle}{L}$ in figure $2(\mathrm{~b})$ is chosen to be 0.75 . This corresponds to $\left|\mathbf{t}_{\perp}\right|^{2} \leq \frac{1}{2}$ in (10) and $\theta_{\max }=\frac{\pi}{4}$ (see also [20]). We estimate that in the above range, the extension is accurate to within $6 \%$. Indeed, Marko and Siggia have shown that the approximation used in (10) gives results that are well within the above error even at much lower values of the extension. All the curves in figure 2(b) are bounded from below by the case of the very long filament $\frac{L}{L_{p}} \rightarrow \infty$ (dashed line). This curve is identical to the one given by Marko and Siggia[17]. It appears that when $\frac{L}{L_{p}}>10$, the filament can be considered to be long with an error smaller than $12 \%$ in the range $0.75<\frac{\left\langle z\left(\frac{L}{2}\right)-z\left(-\frac{L}{2}\right)\right\rangle}{L}<1$. The main point of figure $2(\mathrm{~b})$ is the strong dependence of the force-extension relation on the contour length in the region $\frac{L}{L_{p}}<3$. This prediction is quantitatively consistent with those of Hori et al. [20] who arrived at similar conclusions using path integral techniques. The length dependence of the forceextension relation has also been demonstrated experimentally by Seol et al.[19] for dsDNA, although the contour lengths in their experiment were much larger $\left(\frac{L}{L_{p}}>12.6\right)$. Seol et al. found that the error in determining the persistence length $L_{p}$ by the one parameter fit to the force-extension data, proposed by Marko and Siggia, increased as the contour length became shorter. This is qualitatively consistent with our results. Finally, when $F \rightarrow 0$, the average extension is nearly independent of the force. The curves in figure 2(b) approach the asymptotic value of $1-\frac{1}{6} \frac{L}{L_{p}}$ when $F=0$. 


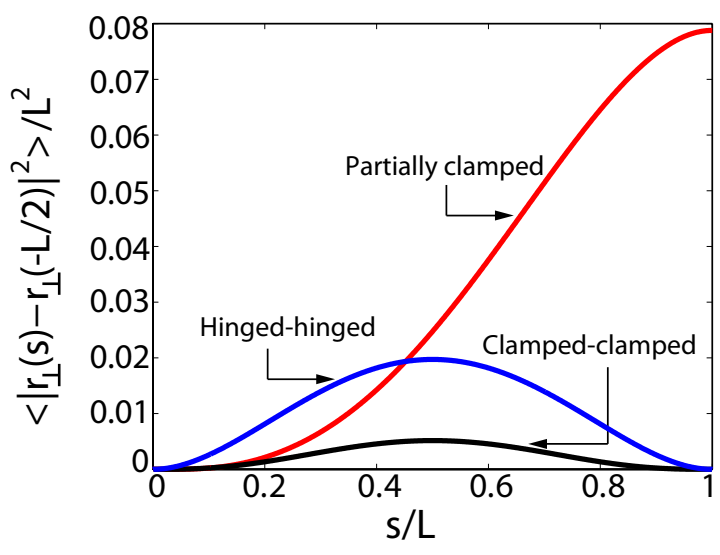

(a)

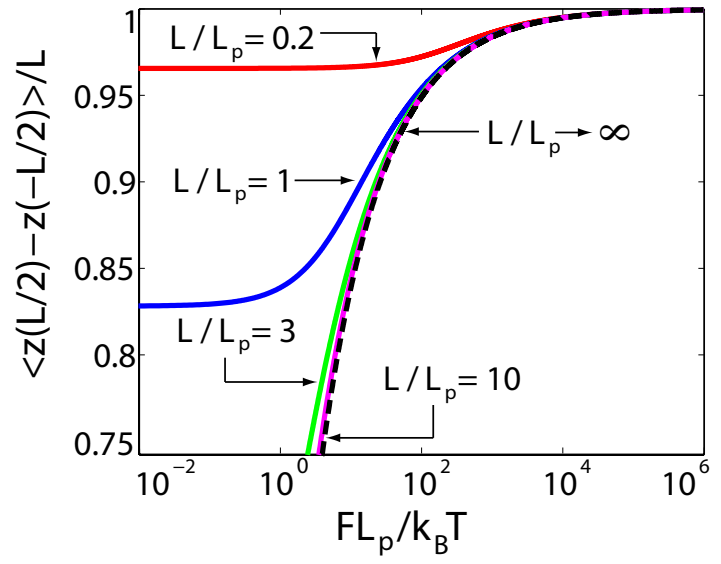

(b)

Figure 2: Contour length and boundary conditions matter in the mechanics of fluctuating rods. (a) The transverse fluctuations of a filamentous macromolecule of non-dimensional length $L / L_{p}=0.5$ at non-dimensional force $F L_{p} / k_{B} T=2.25$ plotted as a function of position along the filament for different boundary conditions. The lateral fluctuations are constrained when both ends are clamped, causing the macromolecule to appear stiffer. (b) Force-extension behavior of a filamentous macromolecule depends strongly on the contour length $L$ of the macromolecule. The length dependence is weak only when the molecule is nearly fully stretched.

\section{Comparison with experiment}

In order to test the validity of our theory, we designed an experiment in which we positioned actin filaments at predetermined locations with the aid of AC electric fields[21] with the goal of observing their transverse fluctuations. During these experiments, we observed that the amplitudes of the filaments' transverse fluctuations depended strongly on the electric field intensity. Our hypothesis was that the electric field altered the tension applied to the filament which in turn determined the amplitude of transverse fluctuations.

Our experimental apparatus consisted of a $75 \mu \mathrm{m}$-high flow cell sandwiched between two glass slides. Using standard microfabrication techniques, a pair of gold electrodes (10nmthick NiCr adhesion layer and $100 \mathrm{~nm}$-thick gold surface layer), with a small gap $G=7 \mu \mathrm{m}$ between them, was patterned on one of the slides, and a $1 \mu \mathrm{m}$-deep trench was etched between the two electrodes to provide free space under the suspended filaments (figure 3(a)). The flow cell was filled with a solution of $50 \mathrm{nM}$ rhodamine-phalloidin-stabilized actin, suspended in $37 \mathrm{mM} \mathrm{KCl,} 2 \mathrm{mM} \mathrm{MgCl}$, $1 \mathrm{mM}$ EGTA, $20 \mathrm{mM}$ Hepes, and $1 \mathrm{mM}$ Dithiothreitol (DTT) (electrical conductivity $0.56 \mathrm{~S} / \mathrm{m}$ ), and was placed on the stage of an inverted microscope. When an $\mathrm{AC}(2 \mathrm{MHz})$ potential difference was applied across the electrodes, the electric field caused the actin to align parallel to the field lines, migrate towards the maximum electric field intensity, and bridge the gap across the electrodes. Figure 3(b) is an image of a single filament spanning the gap between the two electrodes. The bright line corresponds to the fluorescent signal and the asterisks represent the digitized data.

In the absence of electric field, actin filaments occassionally bridged the gap. In these 


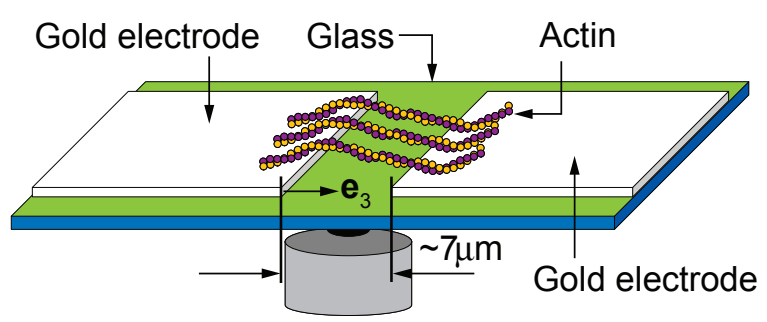

(a)

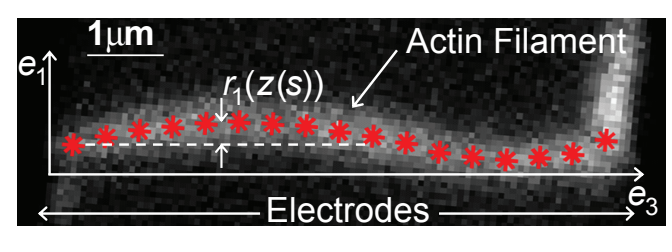

(b)

Figure 3: The measurement of transverse fluctuations. (a) A schematic depiction of the experimental apparatus. (b) Contrast-enhanced image of an actin filament trapped across the gap between two electrodes. The image also depicts the discretized position data $\left(^{*}\right)$. This figure is reproduced from Arsenault et al.[22].

cases the contour lengths $L$ of the filaments' segments confined between the electrodes' edges were typically larger than the gap's width $G$. When the electric field was present, a greater number of filaments bridged the gap between the electrodes, and $L \approx G$. In theory, this provides sufficient information to determine the tension $F$ which satisfies the constraint $\left\langle z\left(\frac{L}{2}\right)-z\left(-\frac{L}{2}\right)\right\rangle=G$ in the force-extension relation (24) for a clamped-clamped filament. However, the contour length $L$ is not accurately known in our experiment, and so a fit relying on (24) is likely to result in large errors. We, therefore, employ (25) to determine the tension since we can accurately measure the magnitude of the transverse fluctuations, as described in the following. Once a filament was observed to bridge the gap across the electrodes, an electron multiplying CCD camera (Photometrics Cascade II) took images at $10 \mathrm{~Hz}$. A custom-written Matlab ${ }^{T M}$ program was used to obtain the component $r_{1}(s)$ (see eqn. (12)) of the filament's transverse displacement (figure 3(b)) as a function of the distance $z(s)$ from one of the anchoring points. The data was then used to compute $\left\langle\left(r_{1}(s)-r_{1}\left(-\frac{L}{2}\right)\right)^{2}\right\rangle=\frac{1}{2}\left\langle\left|\mathbf{r}_{\perp}(s)-\mathbf{r}_{\perp}\left(-\frac{L}{2}\right)\right|^{2}\right\rangle$ as a function of $z(s) / G$. The results for various electric field intensities are plotted in figure $4(\mathrm{a})$ together with the theoretical predictions, (25). In the theory, we used the accepted value of $K_{b} \approx 70 \times 10^{3} \mathrm{pNnm}^{2}$ for actin and determined the tension $F$ that minimizes the discrepancy between theory and experiment. In other words, we use one adjustible parameter. It is immediately apparent that (25) provides an excellent fit to the experimental data. Furthermore, the tension $F$ obtained through the best least-squares fit is found to vary linearly with the square of the electric field, as shown in figure 4(b). This is consistent with the conclusions of Arsenault et al.[22] who fit the same data using a linear Brownian dynamic model of a beam based on the small strain, small rotation assumption. According to this model the equation of motion of an elastic rod moving in a viscous fluid is

$$
\rho \frac{\partial^{2} r_{i}}{\partial t^{2}}+f \frac{\partial r_{i}}{\partial t}=\frac{\partial}{\partial z}\left(F \frac{\partial r_{i}}{\partial z}\right)-K_{b} \frac{\partial^{4} r_{i}}{\partial z^{4}}+W(z, t),
$$

where $i$ can equal 1 or 2 , corresponding to either of the transverse directions, $\rho$ is the linear density, $f$ is the friction coefficient, and $W$ is a white-noise, forcing term. Notice that $s$ is replaced by $z$ in this small strain, small rotation theory. The solution to (30) 


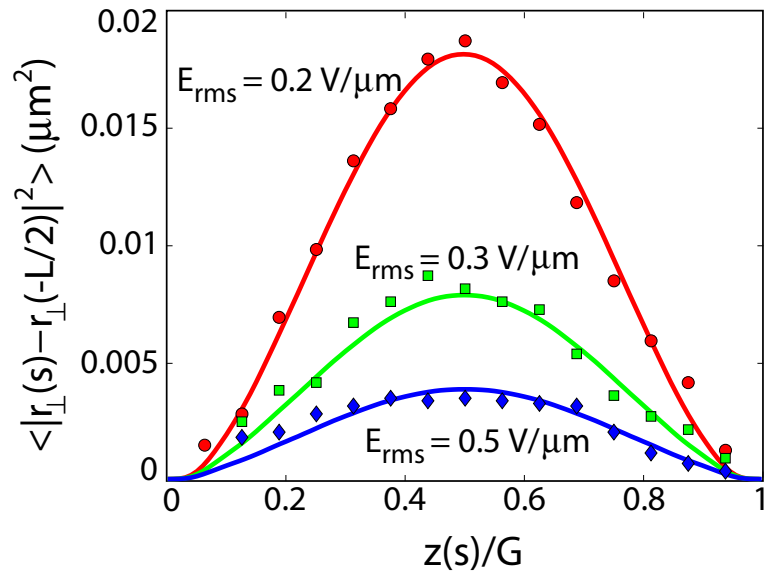

(a)

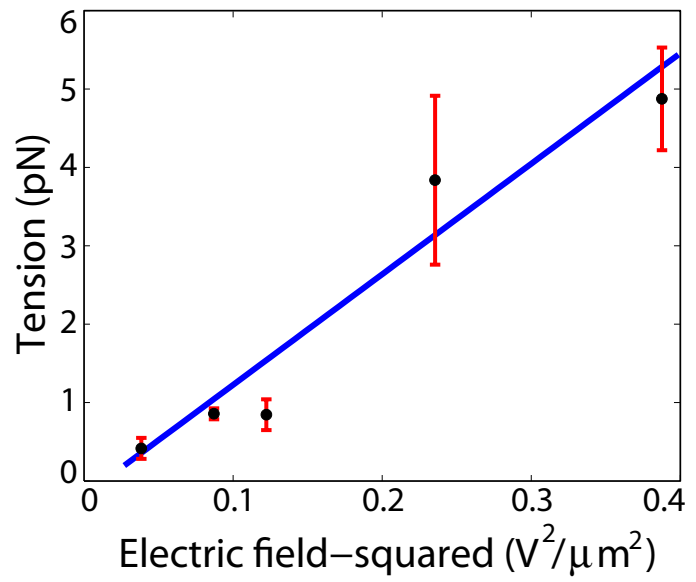

(b)

Figure 4: Validation of theory with experimental data on transverse fluctuations. (a) Experimental data for $\frac{1}{2}\left\langle\left|\mathbf{r}_{\perp}(s)-\mathbf{r}_{\perp}\left(-\frac{L}{2}\right)\right|^{2}\right\rangle$ at three different electric fields (symbols) are fitted to (25) (solid curves) with the tension $F$ as the fitting parameter and a known value of $K_{b}=70 \times 10^{3} \mathrm{pNnm}^{2}$ for actin. (b) The value of $F$ giving the best least-squares fit is found to vary linearly with $E_{r m s}^{2}$ in agreement with the results of a fit using a linear Brownian dynamic model reported in Arsenault et al.[22]. The manner of presentation of the experimental data and the results of the least-squares fit in this figure are similar to that in Arsenault et al.[22] but the data sets are different in each case. 
follows the methods outlined by VanLear and Uhlenbeck[26] and is given in detail in the electronic supplement to Arsenault et al.[22]. The resulting expressions were then fit to the experimental data on the filaments' transverse fluctuation to extract the tension. The tensions predicted by the linear Brownian dynamic model of Arsenault et al. are the same as those of our present theory and the $E_{r m s}^{2}$ dependence of the predicted tension is preserved. In fact, similar values for the tension are recovered when we use the appropriate expression of Hori et al.[20].

\section{Conclusions}

In this paper, we have presented a theory for predicting the extension and variance of transverse fluctuations of inextensible rod-like macromolecules subjected to Brownian motion and large tensile forces. The main new features of the present theory are that (i) it accounts for the contour length dependence of the force-extension relation of the molecule, and (ii) it accomodates a variety of boundary conditions imposed by various testing apparatuses. The present theory recovers the expressions for the force-extension relation given by Marko and Siggia when the contour length $L$ is large relative to the persistence length $L_{p}$. The theory can be used to estimate various mechanical properties of the macromolecules such as flexural rigidity and tension. We have also demonstrated the application of our theory to the transverse motions of a short fluctuating actin filament and found that it provides an excellent fit to the experimental data.

Acknowledgements: We acknowledge partial support from the National Science Foundation through the Nano/Bio Interface Center (NSF NSEC DMR-0425780).

\section{References}

[1] Bhasin N., Carl P., Harper P., Feng G., Lu H., Speicher D. W. and Discher D. E., "Chemistry on a single protein, VCAM-1, during forced unfolding", J. Biol. Chem. 279, 45865-45874, (2004).

[2] Nelson P. Biological Physics, W. H. Freeman and Company, (2004).

[3] Bustamante C., Marko J. F., Siggia E. D. and Smith S., "Entropic elasticity of lambda phage DNA", Science 265, 1599-1600, (1994).

[4] Liphardt J., Onoa B., Smith S. B., Tinoco I. JR and Bustamante C., "Reversible unfolding of single RNA molecules by mechanical force", Science 292, 733-737, (2001).

[5] Abels J. A., Moreno-Herrero F., van der Heijden T., Dekker C. and Dekker N. H., "Single-molecule measurements of persistence length of double-stranded RNA", Biophys. J. 88, 2737-2744, (2005). 
[6] Rief M., Gautel M., Oesterhelt F., Fernandez J.M. and Gaub H. E., "Reversible unfolding of individual titin immunoglobulin domains by AFM", Science 276, 1109-1112, (1997).

[7] Oberhauser A.F., Marszalek P.E., Erickson H.P. and Fernandez J.M., "The molecular elasticity of tenascin, an extracellular matrix protein", Nature, 393, 181-185, (1998).

[8] Law R., Carl P., Harper S., Dalhaimer P., Speicher D. W. and Discher D. E., "Cooperativity in forced unfolding of tandem spectrin repeats", Biophys. J. 84, 533-544, (2003).

[9] Gittes F., Mickey B., Nettleton J. and Howard J., "Flexural rigidity of microtubules and actin filaments measured from thermal fluctuations in shape", $J$. Cell Biology 120(4), 923-934, (1993).

[10] Dupuis D. E., Guilford W. H., Wu J. and Warshaw D. M., "Actin filament mechanics in the laser trap", J. Muscle. Res. Cell Motil. 18, 17-30, (1997).

[11] Gosse C. and Croquette V., "Magnetic tweezers: Micromanipulation and force measurement at the molecular level", Biophys. J 82 3314-3329, (2002).

[12] La Porta A. and Wang M. D., "Optical torque wrench: Angular trapping, rotation, and torque detection of quartz microparticles", Phys. Rev. Lett. 92(19), 190801-190804.

[13] Deufel, C., Forth, S., Simmons C. R., Dejgosha, S. and Wang, M. D., 2007. Nanofabricated quartz cylinders for angular trapping: DNA superocoiling and torque detection. Nature Methods 4(3), 223-225.

[14] Strick, T. R., Allemand, J.-F., Bensimon D., Bensimon A. and Croquette V., 1996. The elasticity of a single supercoiled DNA molecule. Science $\mathbf{2 7 1}$, 18351837.

[15] Moroz, J. D. and Nelson, P. C., 1997. Torsional directed walks, entropic elasticity and DNA twist stiffness. Proc. Natl. Acad. Sci. USA 94 14418-14422.

[16] Fisher T. E., Oberhauser A. F., Carrion-Vazquez M., Marszalek P. E. and Fernandez J. M., "The study of protein mechanics with the atomic force microscope", Trends Biochem. Sci. 24, 379-384, (1999).

[17] Marko, J. F. and Siggia, E. D., 1995. Stretching DNA. Macromolecules 28, 8759-8770.

[18] Odijk, T., 1995. Stiff chains and filaments under tension. Macromolecules 28, 7016-7018.

[19] Seol, Y., Li, J., Nelson P., Perkins, T. T., and Betterton, M. D. 2007. Elasticity of short DNA molecules: theory and experiments for contour lengths of 0.6-0.7 $\mu \mathrm{m}$. To appear in Biophys. J. 
[20] Hori, Y., Prasad, A. and Kondev J., 2007. Stretching short biopolymers by fields and forces. Phys. Rev. E 75, 041904.

[21] Riegelman, M., Liu, H., and Bau, H. H., 2006. Controlled nanoassembly and construction of nanofluidic devices. J. Fluids Eng. 128, 6-13.

[22] Arsenault M. E., Zhao H., Purohit P. K., Goldman Y. E, and Bau H. H., (2007). "Confinement and manipulation of actin filaments by electric fields", Biophys. J. 93, L42-L44.

[23] Moroz, J. D. and Nelson, P. C., 1998. Entropic elasticity of twist storing polymers. Macromolecules 31, 6333-6347.

[24] Sanders J. L., 1963. Nonlinear theories of thin shells. Quat. of Appl. Math. 21(1), 21-36.

[25] O'Reilly, O. M. and Turcotte, J. S., 1997. Elastic rods with moderate rotation. J. of Elasticity 48, 193-216.

[26] Van Lear, G. A. and Uhlenbeck G. E., "The Brownian Motion of Strings and Elastic Rods", Phys. Rev. 38, 1583-1598, (1931).

[27] Nizette, M. and Goriely, A., 1999. Towards a classification of Euler-Kirchhoff filaments. J. Math. Phys. 40(6), 2830-2866. 